\title{
MAXIMUM CYCLE PACKING IN EULERIAN GRAPHS USING LOCAL TRACES
}

\author{
Peter Recht and Eva-Maria Sprengel \\ Operations Research and Business Informatics \\ TU Dortmund \\ D 44221 Dortmund, Germany \\ e-mail: peter.recht@tu-dortmund.de \\ eva-maria.sprengel@tu-dortmund.de
}

\begin{abstract}
For a graph $G=(V, E)$ and a vertex $v \in V$, let $T(v)$ be a local trace at $v$, i.e. $T(v)$ is an Eulerian subgraph of $G$ such that every walk $W(v)$, with start vertex $v$ can be extended to an Eulerian tour in $T(v)$.

We prove that every maximum edge-disjoint cycle packing $\mathcal{Z}^{*}$ of $G$ induces a maximum trace $T(v)$ at $v$ for every $v \in V$. Moreover, if $G$ is Eulerian then sufficient conditions are given that guarantee that the sets of cycles inducing maximum local traces of $G$ also induce a maximum cycle packing of $G$.
\end{abstract}

Keywords: edge-disjoint cycle packing, local traces, extremal problems in graph theory.

2010 Mathematics Subject Classification: 05C38.

\section{REFERENCES}

[1] S. Antonakopulos and L. Zhang, Approximation algorithms for grooming in optical network design, Theoret. Comput. Sci. 412 (2011) 3738-3751.

doi:10.1016/j.tcs.2011.03.034

[2] F. Bäbler, Über eine spezielle Klasse Euler'scher Graphen, Comment. Math. Helv. 27 (1953) 81-100. doi:10.1007/BF02564555

[3] V. Bafna and P.A. Pevzner, Genome rearrangement and sorting by reversals, SIAM J. Comput. 25 (1996) 272-289. doi:10.1137/S0097539793250627 
[4] A. Caprara, Sorting permutations by reversals and Eulerian cycle decompositions, SIAM J. Discrete Math. 12 (1999) 91-110.

doi:10.1137/S089548019731994X

[5] A. Caprara, A. Panconesi and R. Rizzi, Packing cycles in undirected Graphs, J. Algorithms 48 (2003) 239-256.

doi:10.1016/S0196-6774(03)00052-X

[6] G. Fertin, A. Labarre, I. Rusu, É. Tannier and S. Vialette, Combinatorics of Genome Rearrangement (MIT Press, Cambridge, Ma., 2009).

[7] J. Harant, D. Rautenbach, P. Recht and F. Regen, Packing edge-disjoint cycles in graphs and the cyclomatic number, Discrete Math. 310 (2010) 1456-1462. doi:10.1016/j.disc.2009.07.017

[8] J. Harant, D. Rautenbach, P. Recht, I. Schiermeyer and E.-M. Sprengel, Packing disjoint cycles over vertex cuts, Discrete Math. 310 (2010) 1974-1978. doi:10.1016/j.disc.2010.03.009

[9] J. Kececioglu and D. Sankoff, Exact and approximation algorithms for sorting by reversals with application to genome rearrangement, Algorithmica 13 (1997) 180210.

doi:10.1007/BF01188586

[10] M. Krivelevich, Z. Nutov, M.R. Salvatpour, J. Yuster and R. Yuster, Approximation algorithms and hardness results for cycle packing problems, ACM Trans. Algorithm 3(4)) (2007) Article 48.

[11] O. Ore, A problem regarding the tracing of graphs, Elem. Math. 6 (1951) 49-53.

[12] P. Recht and E.-M. Sprengel, Packing Euler graphs with traces, in: Operations Research Proceedings, Klatte, Lüthi and Schmedders (Ed(s)), (Heidelberg, New York, Dordrecht, London, Springer, 2011) 53-58.

Received 29 December 2013 Accepted 10 March 2014 